\title{
Editorial
}

\section{Mouse models for cancer research}

\author{
Wei Zhang, Lynette Moore, and Ping Ji
}

\begin{abstract}
Mouse models of cancer enable researchers to learn about tumor biology in complicated and dynamic physiological systems. Since the development of gene targeting in mice, cancer biologists have been among the most frequent users of transgenic mouse models, which have dramatically increased knowledge about how cancers form and grow. The Chinese Journal of Cancer will publish a series of papers reporting the use of mouse models in studying genetic events in cancer cases. This editorial is an overview of the development and applications of mouse models of cancer and directs the reader to upcoming papers describing the use of these models to be published in coming issues, beginning with three articles in the current issue.
\end{abstract}

Key words Knockout mouse, gene targeting, stem cells, oncogene, tumor suppressor gene

Prior to the development of genetic mouse models, researchers primarily studied cancer using cell culture systems of cell lines derived from human tumors. Although studies using these systems continue to provide valuable information and remain important to cancer research, their limitations include the inability to examine physiological interactions among tumor cells and between tumor cells and their environment in vivo. Use of xenograft models in which human tumor-derived cell lines are implanted in mice overcomes some of these limitations. However, many of such studies must be carried out using immunocompromised mice or subcutaneous implantation, preventing immune responses and site-specific interactions. In an ideal mouse system, one could quickly generate tumors resembling human tumor cells at both the genetic and morphologic levels. Accomplishing this using mouse models has provided an indispensable tool for studying tumor initiation, maintenance, progression, and response to treatment. Currently, a number of genetic manipulation techniques are available to cancer biologists. Selecting a correct strategy for generating mouse models of cancer is an important first step in a research project. Also important is determining the intended purposes of specific mouse models ${ }^{[1,2]}$.

Authors' Affiliation: Department of Pathology, The University of Texas M.D. Anderson Cancer Center, Houston, Texas 77030, USA.

Corresponding Author: Wei Zhang, Department of Pathology, The University of Texas M.D. Anderson Cancer Center, 1515 Holcombe Blvd., Houston, Texas 77030, USA. Tel: +1-713-745-1103; Email: wzhang@mdanderson.org.

\section{Transgenic Mouse Model of Cancer}

Early attempts at inducing expression of cloned genes in mice coincided with the discovery that viral and cellular oncogenes can transform mammalian cells. Thus, determining the ability of these oncogenes to promote tumor formation in vivo would be crucial to increasing understanding of cancer development and progression. Some of the first transgenic mice generated for examining oncogene function were those with expression of the Myc oncogene. Specifically, $E_{\mu-M y c}$ and MMTV-Myc transgenic mice are prone to B-cell lymphomas and mammary tumors, respectively ${ }^{[3,4]}$. These key early studies confirmed the hypothesis that oncogenes can promote tumorigenesis in vivo. In addition, transgenic mice can be crossed with other mouse lines or exposed to environmental chemicals or retroviruses to identify cooperating oncogenes during tumorigenesis.

\section{Gene-Targeting Mouse Model of Cancer}

Delineation of homologous recombination in mammalian cells in the late 1970s and development of elegant-positive and -negative selection methods for accurately identifying the desired genetic events in murine embryonic stem (ES) cells in the 1980s paved the way for modern mouse manipulation. This research has had such an impact on the development of mouse models that in 2007, Mario Capecchi, Oliver Smithies, 
and Martin Evans received the Nobel Prize in Physiology or Medicine for their work in developing technology for introduction of specific mutations of mammalian genes and transferring these mutations to the mouse germline. This technology consists of multiple steps that result in either deletion of the coding sequence of a gene (knockout) or introduction of exogenous sequences into the specific region in the genome (knockin).

Using a knockout approach, the expression of tumor suppressor genes such as $R b$ and $p 53$ as well as candidate tumor suppressor genes can be disrupted to determine their tumor-suppressive functions ${ }^{[5,6]}$. Use of p53-knockout mice in these studies demonstrated that, although unnecessary for mouse embryonic development, loss of one or both alleles of p53 strongly predisposed the animals to cancer. $R b$-heterozygous mice were also predisposed to tumor development, although they did not have retinoblastoma as initially hypothesized, and $R b$-null animals died at early embryonic stages. These knockout studies were pivotal for generation of mouse models for cancer research. However, they had limitations, such as embryonic lethality and less than $100 \%$ accuracy in mimicking the human tumor spectrum.

The effect of gene mutations on physiology can be evaluated using a knockin approach. For example, investigators introduced the $p 53^{R 271 \mathrm{H}}$ mutation into the specific locus of the murine p53 gene to identify the hot-spot mutation frequently observed in patients with cancer $^{[]}$. As they generated more oncomice and knockout mice, the mouse models with individual gene target provided even more information when crossed with mice with various genetic backgrounds. Using different breeding strategies, one can create double knockout mice, or knockout mice that are also transgenic for a given oncogene. These combinations of mice can be used to study molecule interplay, oncogene cooperation, and oncogene-tumor suppressor gene interactions. Furthermore, backcrossed mice can be used to study the effects of different genetic backgrounds on the tumor spectrum in genetically engineered cancer-prone mice. Genetic events in these mouse models can mimic those that are frequently perturbed in human tumors.

\section{Conditional and Inducible Mouse Models of Cancer}

Use of conditional and inducible mouse model systems is valuable for induction of somatic mutations in a tissue-specific and time-controlled manner. The most common conditional system, the Cre-loxP recombination system, is widely used. The relative orientation of the two loxP sites in the DNA sequence determines whether the recombination results in excision, inversion, or translocation of host DNA ${ }^{[8]}$. Cre recombinase mediates site-directed DNA recombination between two 34-bp loxP sequences. This recombinase can be introduced by crossing the Cre-loxP mouse with a mouse from a transgenic line having expression of Cre in a specific tissue or by transducing a lentivirus or adenovirus with the Cre gene into the desired tissues. The benefit of the Cre-loxP conditional system is that it can be used to study gene function in a specific tissue or cell type in a time-dependent manner.

Use of inducible systems allows for temporal control of expression of the gene of interest. For example, the Tet-inducible system (Tet-On/Tet-Off) is mostly used to turn gene expression on and off. This system is composed of the Tet transactivator (tTA)/reverse tTA (rtTA) and the operator sequences of the Tet operon (tetO). In the Tet-On system, rtTA can bind to the tetO site in the promoter region in the presence of tetracycline or doxcycline and then turn on transgene expression. The Tet-Off system works in the opposite way, as tTA binds to tetO sequences and turns on transgene expression only in the absence of tetracycline or doxcycline. Tissue specificity is determined by tissue-specific promoters that drive tTA or rtTA expression ${ }^{[9]}$. Inducible mouse models are very useful in studying the roles of sustained expression of oncogenes such as Ras and IGFBP2 in tumor maintenance.

\section{RNA Interference Mouse Model of Cancer}

The RNA interference (RNAi) mouse model can be used to rapidly silence specific genes using short hairpin RNA (shRNA). Plasmid-based RNAi is commonly used to generate transgenic RNAi mice ${ }^{[10]}$. A plasmid with shRNA can be introduced into ES cells via microinjection, electroporation, or viral infection. The ES cells are subsequently injected into blastocysts or aggregated with tetraploid embryos, and the resulting embryos are then directly transferred into pseudopregnant mice. Tissue-specific shRNA expression can be achieved using the RNA polymerase II promoter. Additionally, using the RNAi approach, the Cre-loxP conditional and Tet-inducible systems can be used to spatiotemporally and reversibly suppress gene activity in vivo. This transgenic RNAi technology is particularly useful for studying gene function in vivo in a relatively high-throughput manner.

\section{Chromosomal Engineering Mouse Model of Cancer}

Use of the chromosomal engineering mouse model is important for recapitulating human chromosomal abnormalities such as large deletions, inversions, and translocations. In this approach, two loxP sites are 
sequentially introduced into two loci in the ES cell genome. The doubly targeted ES cells are then exposed to Cre to induce recombination between the loxP sites and generate the rearranged chromosome. ES cell clones with the rearranged chromosome are then microinjected into blastocysts to generate mutant mice. This approach allows recombination of nonhomologous chromosome segments, thereby creating various chromosomal rearrangements such as deletions, duplications, inversions, and translocations depending on the chromosomal location and relative orientation of the two loxP sites. Using this strategy, researchers have successfully replicated human acute myeloid leukemia with the $\mathrm{t}(8 ; 21)$ translocation. More recently, investigators identified the tumor suppressor gene $C h d 5$ in the human chromosome $1 p 36$, which is frequently deleted in various human cancers ${ }^{[11]}$.

\section{Replication-Competent Avian Sarcoma- Leukosis Virus Long Terminal Repeat with a Splice Acceptor/Avian Subtype A Receptor Mouse Model of Cancer}

More recently, researchers developed the replication-competent avian sarcoma-leukosis virus long terminal repeat with a splice acceptor (RCAS)/avian subtype A receptor (TVA) model of cancer, which uses somatic gene transfer to induce the expression of genes of interest. The strategy used in this model, first developed in the laboratory of Nobel laureate and National Cancer Institute Director Harold Varmus, is induction of the tissue-specific expression of a receptor for the avian subtype A leukosis virus $t v-a^{[12]}$. The $t v-a$ receptor can be placed under the control of a tissue-specific promoter in transgenic mice. RCAS vectors are then used to infect $t v$-a-expressing cells and deliver genes of interest to appropriate tissues. Researchers have used the RCAS/TVA system in several different contexts to create tumor models. Benefits of this model include the ability to study the effects of somatic alterations on gene expression and to study combinations of oncogenes in a single transgenic model, eliminating the use of extensive crossbreeding schemes. The ability to spatially and temporally control gene expression in both oncomice and tumor suppressor knockout mice using this model has provided methods of overcoming some of the early limitations of mouse models described above.

\section{Cancer Metastasis Mouse Model}

Metastasis is a complex process involving a series of steps in which cancer cells leave the original tumor site and migrate to other parts of the body via the bloodstream or the lymphatic system. Because metastasis is a major cause of cancer mortality, it is critical to determine genetic events that are important for metastasis. Two types of mouse models have been highly useful to study metastasis-genetically engineered model and xenograft model systems. Cancer metastasis models driven by the introduction of oncogenic mutations in a tissue-specific manner can faithfully recapitulate important aspects of tumor metastasis. In these models, cancer develops with high penetrance in a stepwise manner, enabling the interrogation of metastasis especially the early steps of metastatic dissemination. In xenograft models, genes can be readily manipulated in vitro in established tumor cells then the altered cells can be introduced into mice to evaluate for effect on metastatic dissemination ${ }^{[13]}$.

\section{Other Application of Mouse Model of Cancer}

Mouse models are extremely useful tools for understanding cancer biology and have provided insights in tumor initiation, progression, and metastasis. Mouse models are also valuable in many other investigations related to cancer, such as the role of environmental factors in tumor development and Anorexia-Cachexia Syndrome (ACS) in patients with advanced cancer. Environmental factors such as hormones, diet, UV, radiation, and chemicals have causal links to specific human cancers. For example, studies on the effects of oophorectomy and estrogen removal led to the discovery that tamoxifen inhibits the growth of mammary tumors in mice. Research on Min mice has shown the influence of dietary fat on polyp phenotype. ACS affects up to $80 \%$ of patients with advanced cancer and has been estimated to be the immediate cause of death in $20 \%$ to $40 \%$ of patients. ACS is often described as the wasting of skeletal muscle, with or without fat loss. Two recent studies studied ACS using mouse models. In one study, the mice engineered to lack myostatin, a member of the transforming growth factor $\beta$ family, exhibit dramatic increases in skeletal muscle mass through the body. The other study investigated ActRIIB, a receptor that control signaling of several ligands in the transforming growth factor $\beta$ family, including myostatin and activin. Many cancers produce a large amount of activin $A$ and overexpression of activin in mice caused severe muscle wasting and ACS. In a mouse model, blocking ActllB signal prevented and reversed the muscle loss that cancer caused ${ }^{[14]}$.

\section{Conclusion}

Taken together, the vast amount of researches over 
the past 3 decades has resulted in the development of powerful mouse models of cancer. These models are the key tools for current and future studies of cancer research. They allow for both the study of normal and aberrant gene interactions in tumors and the recapitulation of human disease in mice, providing a key resource for studies of chemoprevention and for development and testing of novel cancer therapeutics. The current issue of the Chinese Journal of Cancer includes three articles that describe the use of mouse models in studies of glioma, melanoma, and breast cancer metastasis, respectively. The journal will publish additional articles using mouse model in the investigations of other cancers in future issues.

\section{References}

[1] Cheon DJ, Orsulic S. Mouse Models of Cancer [J]. Annu Rev Pathol, 2011,6:95-119.

[2] Walrath JC, Hawes JJ, Van Dyke T, et al. Genetically engineered mouse models in cancer research [J]. Adv Cancer Res, 2010,106:113-164

[3] Adams JM, Harris AW, Pinkert CA, et al. The c-myc oncogene driven by immunoglobulin enhancers induces lymphoid malignancy in transgenic mice [J]. Nature, 1985,318(6046): 533-538.

[4] Stewart TA, Pattengale PK, Leder P. Spontaneous mammary adenocarcinomas in transgenic mice that carry and express MTV/myc fusion genes [J]. Cell, 1984,38(3):627-637.

[5] Donehower LA, Harvey M, Slagle BL, et al. Mice deficient for p53 are developmentally normal but susceptible to spontaneous tumours [J]. Nature, 1992,356(6366):215-221.

[6] Jacks T, Fazeli A, Schmitt EM, et al. Effects of an Rb mutation in the mouse [J]. Nature, 1992,359(6393):295-300.

[7] de Vries A, Flores ER, Miranda B, et al. Targeted point mutations of p53 lead to dominant-negative inhibition of wildtype p53 function [J]. Proc Natl Acad Sci U S A, 2002,99(5):

\section{Acknowledgement}

We would like to thank Don Norwood at the Department of Scientific Publications of MD Anderson Cancer Center for editing of this manuscript. This research is supported in part by the National Institutes of Health through MD Anderson's Cancer Center Support Grant CA016672.

Received 2011-02-12; revised 2011-02-14; accepted 2011-02-16.
2948-2953.

[8] Hoess $\mathrm{RH}$, Wierzbicki A, Abremski $\mathrm{K}$. The role of the loxP spacer region in $\mathrm{P} 1$ site-specific recombination [J]. Nucleic Acids Res, 1986,14(5):2287-2300.

[9] Fisher EM, Lana-Elola E, Watson SD, et al. New approaches for modelling sporadic genetic disease in the mouse [J]. Dis Model Mech, 2009,2(9-10):446-453.

[10] Dickins RA, McJunkin K, Hernando E, et al. Tissue-specific and reversible RNA interference in transgenic mice [J]. Nat Genet, 2007,39(7):914-921.

[11] Yu Y, Bradley A. Engineering chromosomal rearrangements in mice [J]. Nat Rev Genet, 2001,2(10):780-790.

[12] Holland EC, Varmus HE. Basic fibroblast growth factor induces cell migration and proliferation after glia-specific gene transfer in mice [J]. Proc Natl Acad Sci U S A, 1998,95(3):1218-1223.

[13] Bos PD, Nguyen DX, Massague J. Modeling metastasis in the mouse [J]. Curr Opin Pharmacol, 2010,10(5):571-577.

[14] Vanchieri C. Cachexia in cancer: is it treatable at the molecular level? [J]. J Natl Cancer Inst, 2010,102(22):1694-1697.
Submit your next manuscript to Chinese Journal of Cancer and take full advantage of:

- Open access

- No charge to authors

- Quickly published

- Thorough peer review

- Professionally edited

- No space constraints

- Indexed by PubMed, CA, and Google Scholar

Submit your manuscript at

www.cjcsysu.com 